\title{
The extended clearance model and its use for the interpretation of hepatobiliary elimination data
}

Gian Camenisch*,1, Julia Riede ${ }^{1,2}$, Annett Kunze ${ }^{1}$, Jörg Huwyler ${ }^{2}$, Birk Poller ${ }^{1}$ and Kenichi Umehara ${ }^{1}$

${ }^{1}$ Division of Drug Metabolism and Pharmacokinetics, Integrated Drug Disposition Section, Novartis Institutes for BioMedical Research, $\mathrm{CH}-4056$ Basel, Switzerland

${ }^{2}$ Department of Pharmaceutical Sciences, Division of Pharmaceutical Technology, University of Basel, $\mathrm{CH}-4056$ Basel, Switzerland

*Corresponding Author: E-mail: gian.camenisch@novartis.com; Tel.: +41-79-278-58-17; Fax: +41-61-696-85-83

Received: November 14, 2014; Revised: December 18, 2015; Published: March 31, 2015

\begin{abstract}
Hepatic elimination is a function of the interplay between different processes such as sinusoidal uptake, intracellular metabolism, canalicular (biliary) secretion, and sinusoidal efflux. In this review, we outline how drugs can be classified according to their in vitro determined clearance mechanisms using the extended clearance model as a reference. The approach enables the determination of the ratedetermining hepatic clearance step. Some successful applications will be highlighted, together with a discussion on the major consequences for the pharmacokinetics and the drug-drug interaction potential of drugs. Special emphasize is put on the role of passive permeability and active transport processes in hepatic elimination.
\end{abstract}

\section{Keywords}

extended clearance concept classification system; hepatic elimination; passive permeability; transporters

\section{Extended clearance model}

Historically, hepatic clearance models assumed that (i) the unbound drug concentration in blood is determining the hepatic clearance (metabolism and/or biliary excretion) and that (ii) there is no membrane transport barrier limiting access to the enzymes or transporters in the hepatocyte. Improved models, however, reflect the physiological reality more precisely. In the liver drugs first have to overcome the membrane barrier separating the blood in the sinusoid from the cytosol of the hepatocytes. Permeation across this barrier might occur by passive diffusion and/or active carrier-mediated transport. Once in the cytosol drugs are subject to metabolism, efflux transporter-mediated canalicular (biliary) secretion and/or back-flux (active or passive) into the sinusoid. Consequently, generally referred as the extended clearance model $(E C M)$, the overall hepatic intrinsic drug clearance $\left(\mathrm{CL}_{h, \text { int }}\right)$ can be described as the interplay between all these processes as follows $[1,2]$ :

$C L_{h, \text { int }}=\frac{P S_{\text {inf }} \cdot C L_{i n t}}{P S_{\text {eff }}+C L_{i n t}}=\frac{\left(P S_{\text {inf }, a c t}+P S_{\text {inf }, \text { pas }}\right) \cdot\left(C L_{i n t, m e t}+C L_{\text {int }, \text { sec }}\right)}{P S_{\text {eff }, a c t}+P S_{\text {eff }, \text { pas }}+C L_{\text {int }, \text { met }}+C L_{\text {int }, \text { sec }}}$ 
where, $\mathrm{PS}_{\text {inf,act }}$ and $\mathrm{PS}_{\text {inf,pas }}$ are the active and passive hepatic influx clearances from the blood, respectively, $\mathrm{CL}_{\text {int,sec }}$ is the intrinsic biliary secretion clearance, and $\mathrm{CL}_{\text {int,met }}$ is the intrinsic metabolic clearance. $\mathrm{PS}_{\text {eff,act }}$ and $\mathrm{PS}_{\text {eff,pas }}$ describe the active and passive sinusoidal efflux from the hepatocytes back into the blood, respectively. $P S_{\text {inf }}$ is the sum of $P S_{\text {inf,act }}$ and $P S_{\text {inf,pas, }} \mathrm{PS}_{\text {eff }}$ is the sum of $P S_{\text {eff,act }}$ and $P S_{\text {eff,pas }}, \mathrm{CL}_{\text {int }}$ is the sum of $\mathrm{CL}_{\text {int,sec }}$ and $\mathrm{CL}_{\text {int,met }}$ and $\mathrm{CL}_{\text {int, }}$ is the overall intrinsic hepatic clearance.

The extended clearance model allows the identification of the rate-determining hepatic clearance step for a given drug molecule [1]. Depending on the relative contributions of the individual processes in Eq. (1), four different cases can be distinguished (Fig. 1). While (passive) hepatic uptake is the rate-determining step for ECM class 1 compounds, the sum of metabolism and efflux transporter-mediated biliary elimination is predicted to be the rate-limiting step for ECM class 2 compounds. The overall hepatic uptake (sum of active and passive) is projected to be rate-determining for ECM class 3 compounds, whereas the overall intrinsic clearance of ECM class 4 compounds is dependent on the interplay of all processes involved in hepatic elimination (namely metabolism, uptake, and efflux).

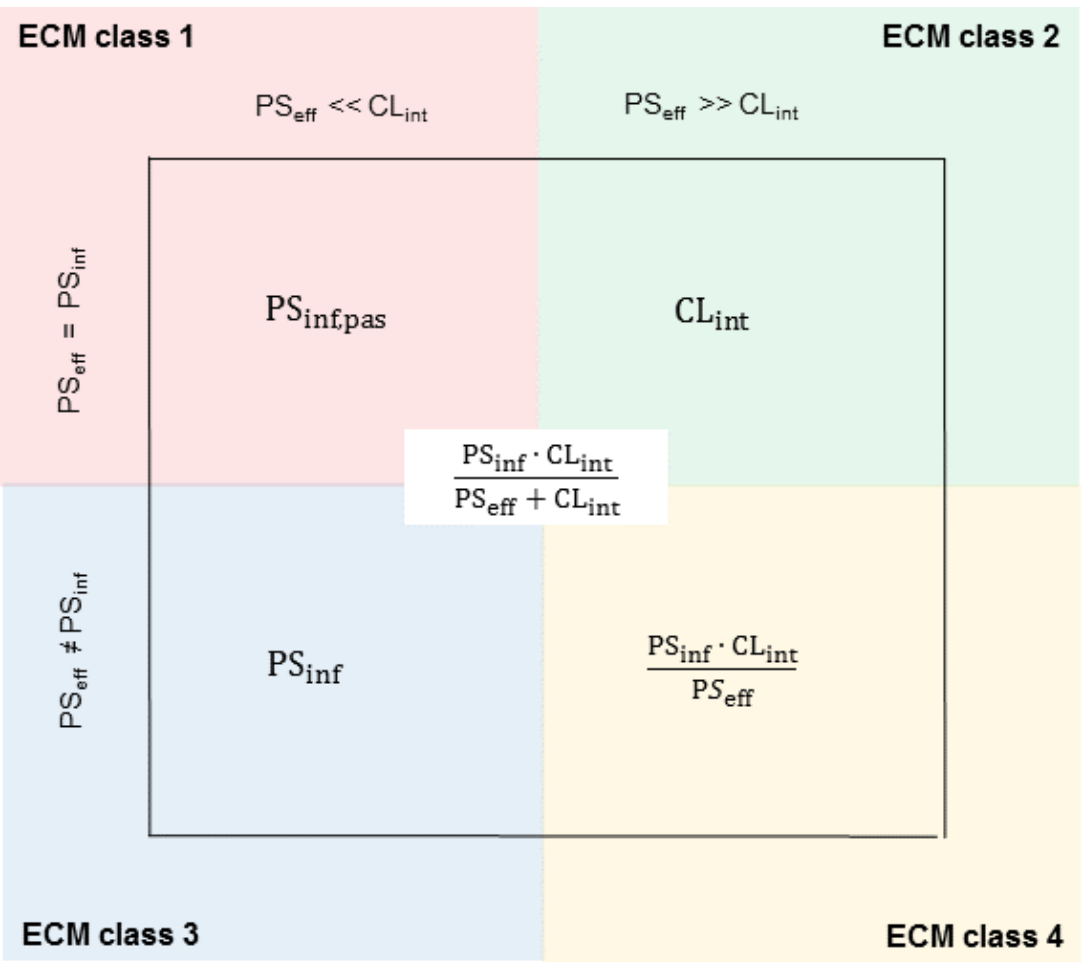

Figure 1. Rate-determining hepatic clearance processes (inner panels) derived from the extended clearance model (ECM) based on different pre-requisites (outer panels) and the assumption thyat $\mathrm{PS}_{\text {eff,pas }}$ is equal to $\mathrm{PS}_{\text {inf,pas }}$ and that $\mathrm{PS}_{\text {eff,act }}$ equals zero (Eq. (1)).

Viewing the liver as a single compartment and assuming that drug molecules are distributing instantaneously and homogeneously within the liver upon entering (well-stirred liver model) the hepatic blood clearance $\left(\mathrm{CL}_{h}\right)$ can be calculated with:

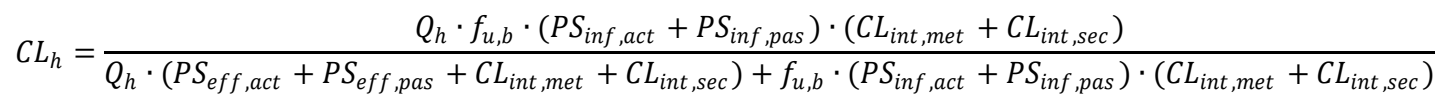


where $Q_{\mathrm{h}}$ is the hepatic blood flow $(20.7 \mathrm{~mL} /(\min \cdot \mathrm{kg}))$ and $f_{\mathrm{u}, \mathrm{b}}$ is the unbound fraction in blood. The clearance parameters of the extended clearance model can be determined experimentally as discussed in full detail elsewhere [1,3]. In brief, $\mathrm{PS}_{\text {inf,act }}$ and $\mathrm{PS}_{\text {inf,pas }}$ can be assessed by uptake experiments in suspended human hepatocytes $(\mathrm{SHH}), \mathrm{CL}_{\text {int,sec }}$ can be determined in human sandwich-cultured hepatocyte incubations (generally assuming that metabolism in this system is negligible) whereas $\mathrm{CL}_{\text {int,met, }}$, assuming absence of non-oxidative metabolism, is usually experimentally evaluated in human liver microsomal (HLM) incubations. Efflux over the sinusoidal membrane from hepatocytes back into the blood is frequently assumed to occur via passive diffusion only (i.e. $\mathrm{PS}_{\text {eff,act }}=0$ ). The experimental determination of this parameter is difficult. In the absence of measured data, the passive sinusoidal efflux is usually assumed to be equal to the passive influx (i.e. $P S_{\text {eff,pas }}=P S_{\text {inf,pas }}$ ). The individual clearances can finally be fed into Eq. (1) and Eq. (2) to predict $C L_{h, \text { int }}$ and $C L_{h}$ of a drug. Table 1 provides a compilation of experimentally determined intrinsic clearances for a dataset of 19 physicochemically diverse compounds from the literature together with corresponding in vivo reference data and the ECM classification according to Fig. 1 [1, 4-7].

Table 1. Experimental human hepatic process clearances from in vitro assays and corresponding in vivo reference data.

\begin{tabular}{|c|c|c|c|c|c|c|c|c|c|c|}
\hline \multirow[b]{2}{*}{ compounds } & \multicolumn{7}{|c|}{ Experimental in vitro data } & \multicolumn{3}{|c|}{ In vivo reference data } \\
\hline & $\begin{array}{c}\text { ECM/ECC } \\
-\end{array}$ & $\begin{array}{l}\mathrm{PS}_{\text {inf,act }} \\
\mathrm{mL} /(\min \cdot \mathrm{kg})\end{array}$ & $\begin{array}{c}\mathrm{PS}_{\text {eff }}= \\
\mathrm{PS}_{\text {inf.pas }} \\
\mathrm{mL} /(\min \cdot \mathrm{kg})\end{array}$ & $\begin{array}{l}\mathrm{CL}_{\text {int, met, } \mathrm{HLM}} \\
\mathrm{mL} /(\min \cdot \mathrm{kg})\end{array}$ & $\begin{array}{l}\mathrm{CL}_{\text {int,met, } \mathrm{HH}} \\
\mathrm{mL} /(\min \cdot \mathrm{kg})\end{array}$ & $\begin{array}{c}\mathrm{CL}_{\text {int,sec }} \\
\mathrm{mL} /(\min \cdot \mathrm{kg})\end{array}$ & $\begin{array}{l}f_{u, b} \\
-\end{array}$ & $\begin{array}{c}\mathrm{CL}_{\mathrm{h}, \mathrm{obs}} \\
\mathrm{mL} /(\min \cdot \mathrm{kg})\end{array}$ & $\begin{array}{c}\mathrm{fn}_{\text {ren }} \\
-\end{array}$ & $\begin{array}{c}f n_{m e t} \\
-\end{array}$ \\
\hline lovastatin acid & $3 / 1$ & 165.1 & 145.5 & 459.0 & 119.6 & 0.0 & 0.08 & 11.4 & 0.10 & 0.90 \\
\hline simvastatin acid & $3 / 1$ & 116.1 & 297.9 & 769.2 & ND & 1.7 & 0.11 & 25.2 & 0.13 & 0.87 \\
\hline propanolol & $4 / 2$ & 300.7 & 276.3 & 110.8 & 29.2 & 6.8 & 0.11 & 12.8 & 0.01 & 0.99 \\
\hline quinidine & $4 / 2$ & 229.4 & 109.3 & 28.4 & 18.0 & 5.1 & 0.27 & 8.7 & 0.19 & 0.81 \\
\hline verapamil & $2 / 2$ & 0.0 & 258.2 & 127.7 & 33.4 & 8.1 & 0.13 & 13.7 & 0.03 & 0.97 \\
\hline ketoconazole & $2 / 2$ & 0.0 & 1568.5 & 97.4 & ND & 29.6 & 0.02 & 3.9 & 0.03 & 0.97 \\
\hline cerivastatin & $4 / 2$ & 221.5 & 243.8 & 46.9 & ND & 0.0 & 0.02 & 3.4 & 0.00 & 1.00 \\
\hline fluvastatin & $4 / 2$ & 218.7 & 325.5 & 146.8 & ND & 0.0 & 0.04 & 7.0 & 0.00 & 1.00 \\
\hline pitavastatin & $4 / 2$ & 364.3 & 258.7 & 17.7 & ND & 0.0 & 0.07 & 3.5 & 0.00 & ND \\
\hline aliskiren & $3 / 3$ & 32.3 & 25.4 & 89.2 & ND & 31.2 & 0.70 & 11.3 & 0.25 & 0.10 \\
\hline cimetidine & $3 / 3$ & 3.0 & 3.6 & 528.7 & 3.2 & 0.2 & 0.84 & 2.7 & 0.84 & 0.14 \\
\hline digoxin & $3 / 3$ & 20.0 & 6.9 & 24.2 & ND & 18.4 & 0.82 & 4.6 & 0.66 & 0.04 \\
\hline cyclospsorine A & $3 / 4$ & 113.2 & 41.9 & 77.6 & 13.5 & 9.1 & 0.03 & 3.1 & 0.01 & 0.96 \\
\hline atorvastatin & $4 / 4$ & 140.4 & 57.7 & 64.6 & 254.4 & 11.8 & 0.08 & 5.9 & 0.02 & 0.69 \\
\hline furosemide & $4 / 4$ & 11.1 & 23.9 & 19.0 & 0.9 & 1.2 & 0.03 & 0.4 & 0.66 & 0.01 \\
\hline ciprofloxacin & $4 / 4$ & 7.0 & 22.9 & 22.0 & ND & 0.0 & 0.69 & 4.5 & 0.60 & 0.12 \\
\hline valsartan & $4 / 4$ & 16.0 & 18.5 & 4.1 & ND & 21.5 & 0.09 & 0.6 & 0.29 & 0.11 \\
\hline pravastatin & $4 / 4$ & 57.9 & 36.0 & 0.9 & 5.3 & 2.2 & 0.97 & 10.4 & 0.47 & 0.30 \\
\hline rosuvastatin & $4 / 4$ & 27.2 & 24.8 & 1.5 & ND & 5.7 & 0.17 & ND & 0.30 & 0.10 \\
\hline
\end{tabular}

ND: Not determined or experimental data subject to a high degree of uncertainty as discussed elsewhere [4]. With exception of the hepatocyte turn-over data $[5,6]$ all hepatic in vitro and in vivo data were taken from previous in-house manuscripts $[1,4,7]$. ECM and ECC class assignment was performed according to Fig. 1 and Fig. 6, respectively (underlying working principle: class 1 or 3 if $2 \cdot$ PS $_{\text {eff }}<$ $\left(\mathrm{CL}_{\text {int,met,HLM }}+\mathrm{CL}_{\text {int,sec }}\right)$, otherwise class 2 or 4$)$. Metabolic clearance data from human liver microsomes and human hepatocytes are labeled with the subscripts $\mathrm{HLM}$ and $\mathrm{HH}$, respectively. $\mathrm{fn}_{\text {met }}\left(=\mathrm{CL}_{\text {met,obs }} / \mathrm{CL}_{\text {tot,obs }}\right)$ and $\mathrm{f} \mathrm{n}_{\text {ren }}\left(=\mathrm{CL}_{\text {ren,obs }} / \mathrm{CL}_{\text {tot,obs }}\right)$ values were calculated from the observed total $\left(\mathrm{CL}_{\text {tot,obs }}\right)$, renal $\left(\mathrm{CL}_{\text {ren,obs }}\right)$ and metabolic $\left(\mathrm{CL}_{\text {met,obs }}\right)$ clearances as derived from peroral human mass balance studies taking into consideration the estimated absolute oral bioavailability $(F)$. Thereof, $f n_{h}\left(=1-f n_{\text {ren }}\right)$ and $f n_{\text {sec }}\left(=f n_{h}-f n_{\text {met }}\right)$ values discussed in this manuscript can be calculated. 


\section{Role of membrane permeability in hepatic elimination}

The likelihood that drugs will be subject to enzyme and/or canalicular efflux transporter activities in the liver depends on the extent of their sinusoidal membrane permeation. For the compounds in our dataset (Table 1) this is illustrated in Fig. 2 depicting the relationship between (in vitro) sinusoidal passive uptake $\left(P S_{\text {inf,pas }}\right)$ and the fractional contribution of metabolism ( $f n_{\text {met }}$, panel $A$ ) or biliary secretion $\left(f n_{\text {sec, }}\right.$ panel $B$ ) to overall in vivo elimination.
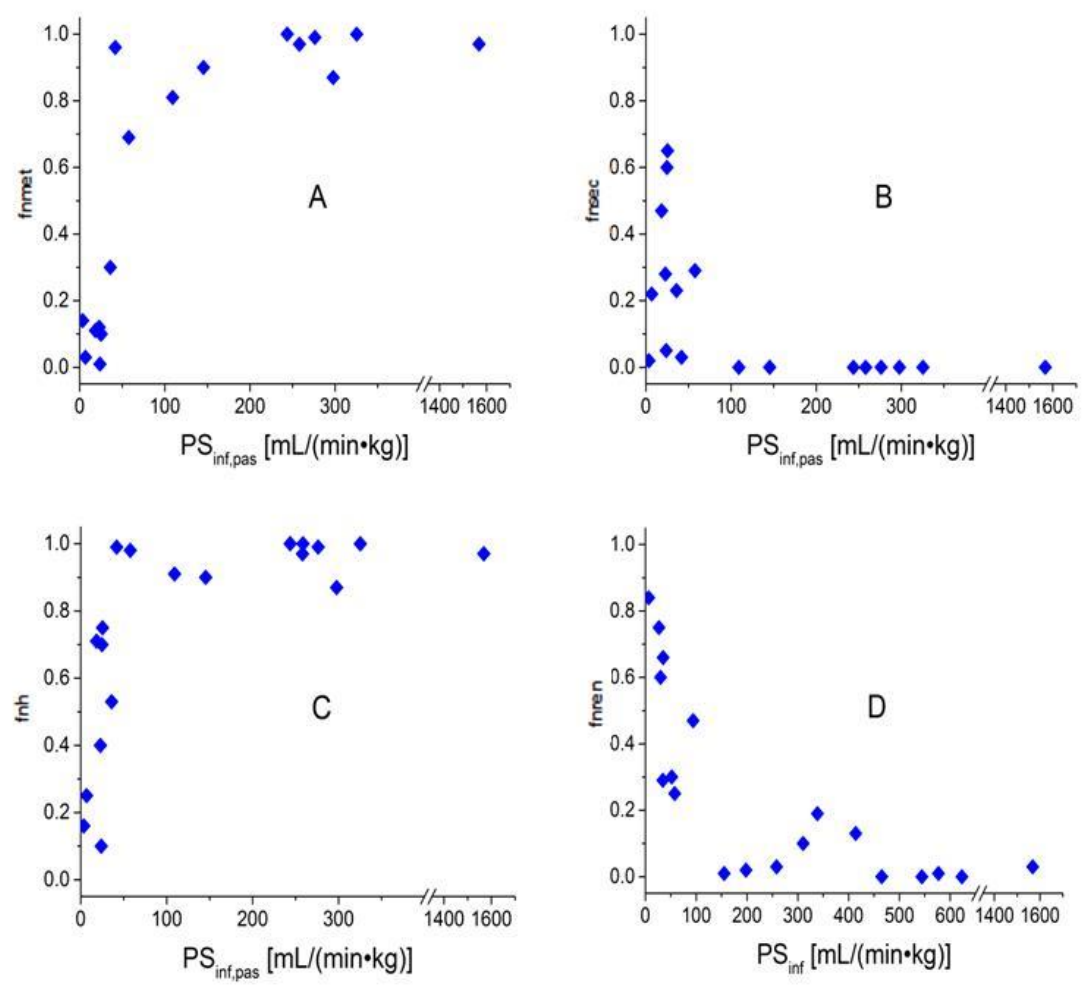

Figure 2. Correlation of sinusoidal hepatic uptake clearances $\left(\mathrm{PS}_{\text {inf,pas }}\right.$ or $\left.\mathrm{PS}_{\text {inf }}\right)$ with the fractional contributions of metabolic (panel A), biliary (panel B), hepatic (panel C) and renal (panel D) clearance to overall in vivo elimination.

An increase in lipophilicity is well known to promote the passive permeability potential of drugs as well as their affinity to drug metabolizing enzymes [8]. Above observed inter-relationship, as depicted in Fig. 2 (panel A), is therefore not very surprising. The association of permeability and enzyme activity allows to define an approximate sinusoidal permeability threshold above which drug elimination seems predominantly driven by metabolism ( $200 \mathrm{~mL} /(\mathrm{min} \cdot \mathrm{kg})$ ). The corresponding plot of $P S_{\text {inf,pas }} \mathrm{vs}^{\mathrm{fn}} \mathrm{f}_{\text {sec }}$ reveals that compound recognition by canalicular efflux transporters, in contrast to $\mathrm{fn}_{\text {met }}$, is by some means inversely correlated with rising sinusoidal permeability (Fig. 2, panel B). Fig. 3 shows the contribution of the measured in vivo metabolic clearance to the in vivo hepatic clearance $\left(=f n_{m e t} / f n_{h}\right)$ for the compounds in our dataset. Not astonishingly, the compounds with a high (total) sinusoidal permeability are predominantly cleared via hepatic metabolism, while the contribution of metabolism generally decreases for lower permeability compounds apparently accompanied by an increasing contribution of active canalicular secretion. 


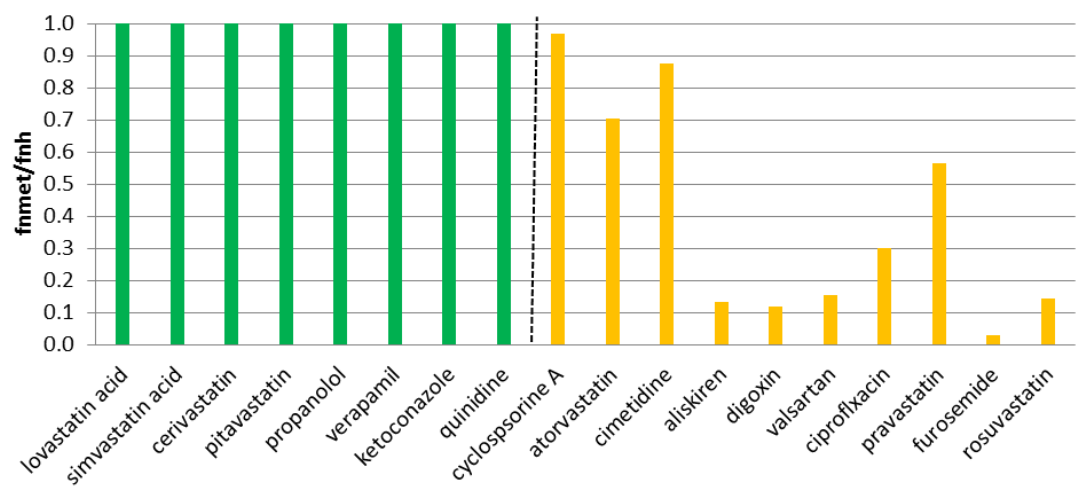

Figure 3. Contribution of in vivo metabolism to overall hepatic clearance for the dataset in Table 1. The dotted line separates the high permeability compounds (green bars) from the low permeability drugs (orange bars) as determined by uptake experiments in suspended hepatocytes $\left(\mathrm{PS}_{\text {inf }}\right.$ threshold: $\left.200 \mathrm{~mL} /(\mathrm{min} \cdot \mathrm{kg})\right)$.

\section{Hepatic versus non-hepatic elimination}

Figure 2 (panel C) illustrates the relationship between passive sinusoidal uptake $\left(P S_{\text {inf,pas }}\right)$ and the observed (in vivo) fractional contribution of hepatic clearance to overall drug elimination $\mathrm{fn}_{\mathrm{h}}$. From this illustration it appears that a sinusoidal permeability of above $40-60 \mathrm{~mL} /(\mathrm{min} \cdot \mathrm{kg})$ will result in predominant hepatic clearance (> $80 \%$ ). Numerous data demonstrates that permeability also exerts a key role in renal elimination similar to the one discussed here for liver [4]. It could be demonstrated that for the highly permeable compounds, despite significant tubular secretion, reabsorption from the tubule back into the blood is so extensive that overall renal clearance for these compounds will be low. For the low permeable compounds on the other hand reabsorption, in agreement with their reduced permeability potential, will be much less. As illustrated in Fig. 4, for all compounds in our dataset with a (passive) sinusoidal permeability beyond $40 \mathrm{~mL} /(\mathrm{min} \cdot \mathrm{kg})$ renal clearance is a minor route of elimination $\left(\mathrm{fn}_{\text {ren }}<20 \%\right)$. For the lower permeable compounds the fractional contribution of renal clearance to total body clearance exceeds $20 \%$ or even represents the predominant route of elimination suggesting that hepatic and renal elimination are somehow complementary with regards to the role of sinusoidal permeability in drug elimination.

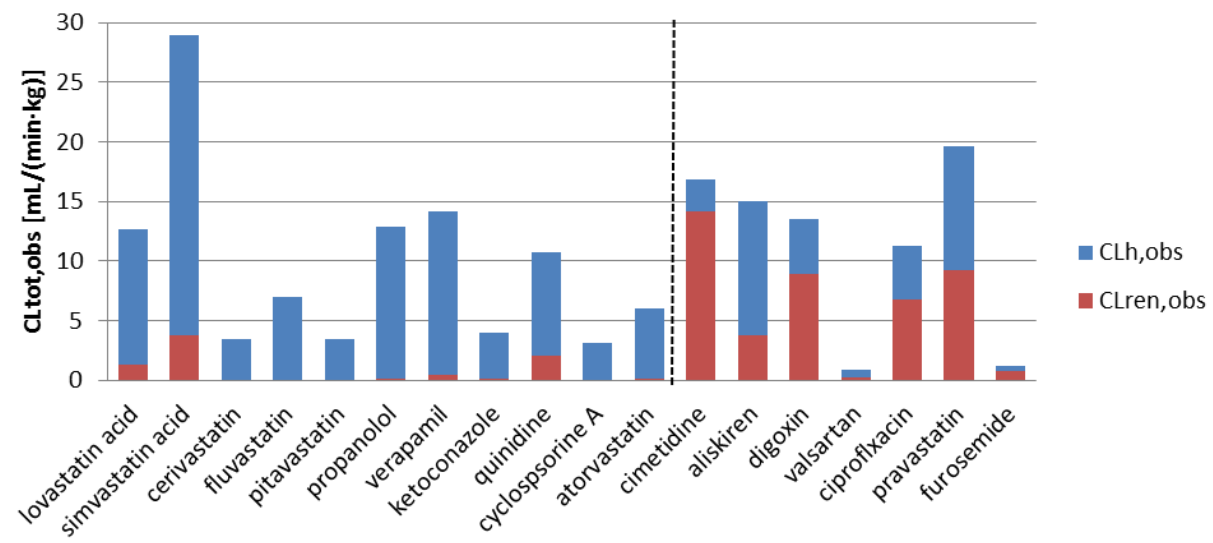

Figure 4. Contributions of the observed renal (red bars) and hepatic (blue bars) clearance pathways to the total body clearance $\left(\mathrm{CL}_{\text {tot,obs }}=\mathrm{CL}_{\mathrm{h}, \mathrm{obs}}+\mathrm{CL}_{\text {ren,obs }}\right)$ for the compounds in Table 1. The dotted line separates the compounds with a $\mathrm{PS}_{\text {inf,pas }}$ value greater (left part) or lower (right part) than $40 \mathrm{~mL} /(\min \cdot \mathrm{kg})$ ). 
Fig. 2 (panel D) illustrates the relationship between $f n_{\text {ren }}$ and total sinusoidal uptake $\left(P S_{\text {inf }}\right)$ for the present dataset. As discussed above, a low permeability is hindering hepatic elimination pushing drugs towards alternative elimination routes (with decreasing lipophilicity plasma protein binding usually decreases which would result in increased glomerular filtration process, possibly increased active tubular secretion and less reabsorption) [4]. Beyond a $\mathrm{PS}_{\text {inf }}$ value of $100 \mathrm{~mL} /(\mathrm{min} \cdot \mathrm{kg})$ ) renal elimination becomes very unlikely. Consequently, following integration of the information from this and the previous chapter, it becomes apparent that total sinusoidal uptake is THE predominant gatekeeper between almost exclusive metabolic hepatic elimination (> $200 \mathrm{~mL} /(\min \cdot \mathrm{kg})$ ), mixed hepatic (metabolism plus biliary secretion, $100-200 \mathrm{~mL} /(\mathrm{min} \cdot \mathrm{kg}))$ and mixed hepatic/renal elimination (metabolism plus biliary and urinary secretion, $<100 \mathrm{~mL} /(\mathrm{min} \cdot \mathrm{kg})$ ) (Fig. 5). Exclusive renal clearance is expected only if $\mathrm{PS}_{\text {inf }}$ is approaching a very low value or if substantial entero-hepatic circulation is taking place. Yet, renal excretion might become the predominant elimination pathway for low permeable compounds with about $\mathrm{PS}_{\text {inf }}<30 \mathrm{~mL} /(\mathrm{min} \cdot \mathrm{kg}$ ) (Fig. 2, panel D). For compounds with sinusoidal uptake between $30-60 \mathrm{~mL} /(\mathrm{min} \cdot \mathrm{kg})$, mixed urinary/biliary elimination seems to be the most probable clearance pathway. Consequently, the relationship between $\mathrm{PS}_{\text {inf,pas }}$ and $\mathrm{fn}_{\mathrm{sec}}$ as shown in Figure 2 (panel B) likely needs to be interpreted as a bell-shaped curve with a $\mathrm{PS}_{\text {inf,pas }}$ maximum at around $30 \mathrm{~mL} /(\mathrm{min} \cdot \mathrm{kg})\left(\right.$ maximal $\left._{\mathrm{fn}} \mathrm{sec}_{\mathrm{sec}} \approx 0.6-0.7\right)$.

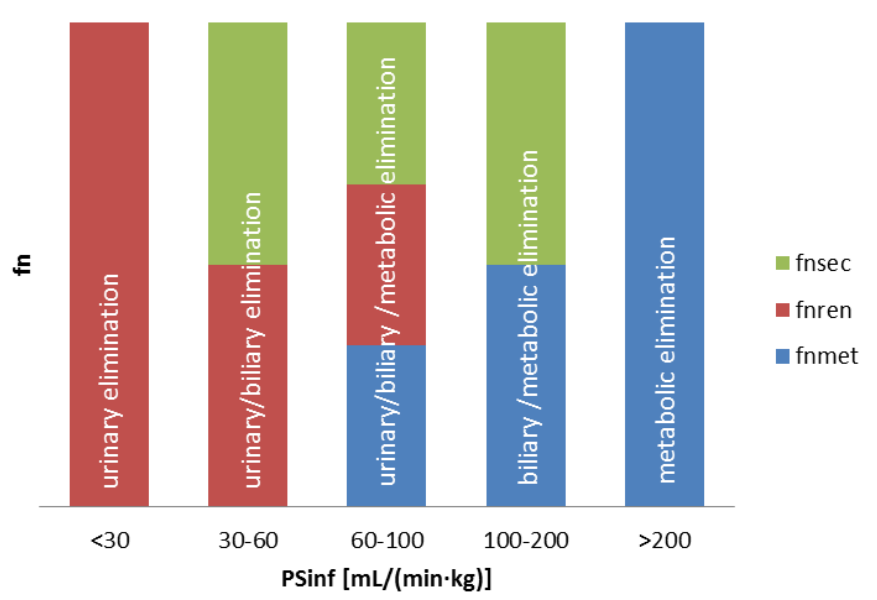

Figure 5. Schematic illustration of the change in elimination route as a function of sinusoidal uptake $\left(P S_{\text {inf }}\right) . f n_{\text {met }}, f n_{\text {ren }}$ and $\mathrm{fn}_{\mathrm{sec}}$ denote the fractional contributions of (hepatic) metabolic, urinary and biliary elimination to overall clearance, respectively. Above a $\mathrm{PS}_{\text {inf }}$ threshold of about $60 \mathrm{~mL} /(\mathrm{min} \cdot \mathrm{kg})$ liver is the expected primary clearance organ whereas below $30 \mathrm{~mL} /(\mathrm{min} \cdot \mathrm{kg})$ renal elimination is likely predominant.

\section{Extended Clearance Concept Classification System}

The extended clearance concept (ECC) is the intangible attempt to complete the principles of the extended clearance model (Fig. 1) with the gatekeeping role of sinusoidal influx as discussed above (Fig. 5) to allow prediction of in vivo pharmacokinetic performance of drugs from in vitro permeability and metabolism data as illustrated in Fig. 6 . Drugs with a high apparent hepatic intrinsic clearance are removed from the blood essentially as fast as they can be delivered to the liver, i.e. independent of protein binding and intrinsic enzyme and canalicular efflux transporter activities. Therefore, the elimination of such drugs is highly dependent upon liver blood flow and the inherent ability to cross the sinusoidal membrane rapidly. Consequently, according to the well-stirred liver model, a compound can be ranked as highly permeable if 
$\mathrm{CL}_{h, \text { int }}=P \mathrm{PS}_{\text {inf,pas }}>\mathrm{Q}_{\mathrm{h}}$ (Eq. (1) and Eq. (2)). From above discussion it is evident that a (passive) sinusoidal permeability above $60 \mathrm{~mL} /(\mathrm{min} \cdot \mathrm{kg}$ ) results in an almost complete hepatic clearance (Fig. 2, panel $\mathrm{C}$ and Fig. 5). Thus, a compound can be ranked as highly permeable if $P S_{\text {inf,pas }} \geq 3$-fold $Q_{h}$, a threshold substantiating the conceptual approximation $\mathrm{PS}_{\text {inf }} \approx \mathrm{PS}_{\text {eff }} \approx \mathrm{PS}_{\text {inf,pas }}$ pertinent to all compounds identified to be hepatic uptake transporter substrates besides demonstrating a high intrinsic permeation potential across the sinusoidal membrane of hepatocytes. Hence, it becomes evident that uptake transporter effects for highly permeable compounds are minimally contributing to overall hepatic clearance while they are expected to become important/predominant for the hepatic clearance of lower permeable compounds. Applying this fundamental relationship, the 19 compounds in our dataset were assigned to the four different ECC classes as summarized in Table 1.

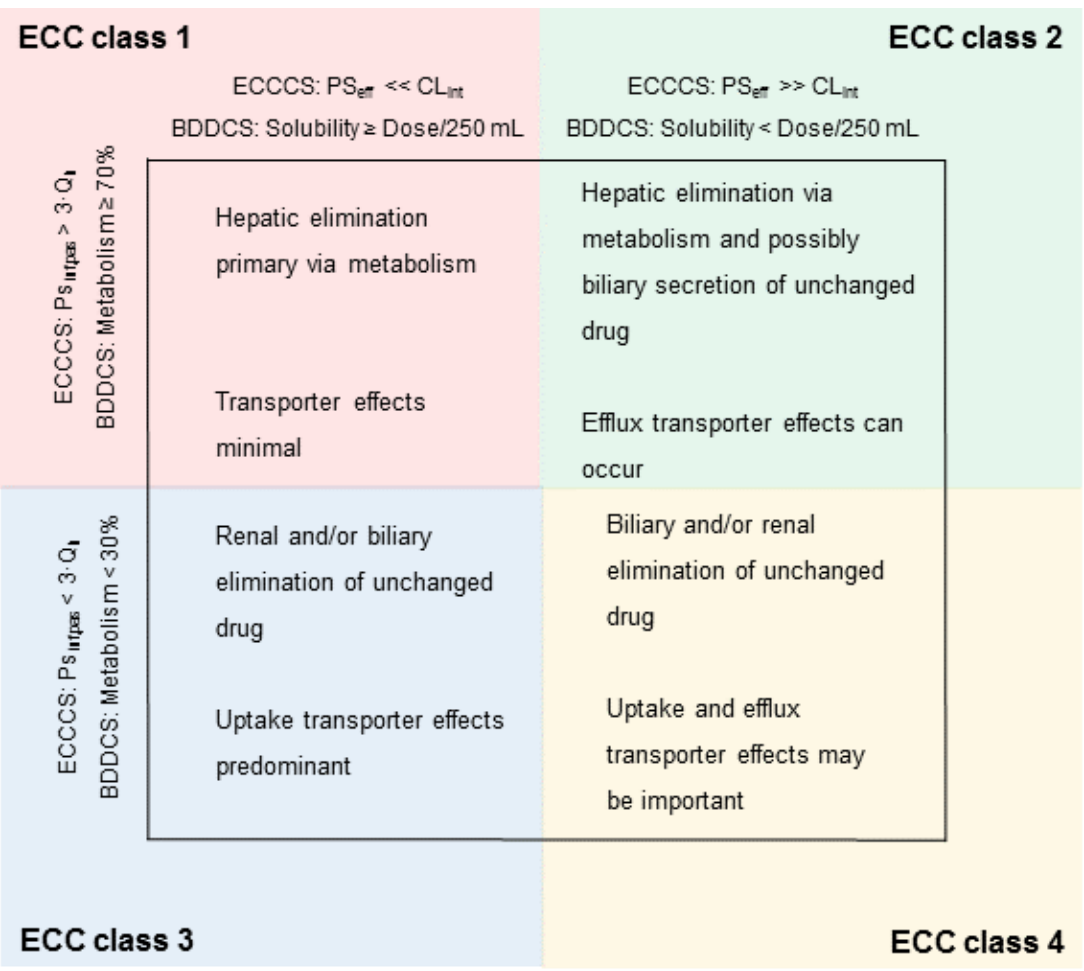

Figure 6. Predominant routes of drug elimination and potential transporter effects on (hepatic) drug disposition according to the Extended Clearance Concept Classification System (ECCCS). Thresholds as defined by the Biopharmaceutics Drug Disposition Classification system (BDDCS) are provided for comparative reasons also $[1,8]$.

Taking all above principles into account it is appropriate to conclude that the major route of elimination for ECC class 3 and 4 compounds is renal and biliary excretion of unchanged drug whereas metabolism is the predominant elimination pathway for ECC class 1 and 2 compounds as previously concluded by others also [8]. However, ECC allows a compound classification of drug molecules based on their in vitro determined (hepatic) clearance parameters in contrast to the Biopharmaceutics Drug Disposition Classification System (BDDCS) which allocates drugs into four classes according to their in vivo metabolism and solubility potential. Both concepts are closely related and have in common that they recognize that the fundamental parameter controlling (hepatic) drug disposition is the compound class-dependent interplay between transporters, enzymes and membrane permeability. Both systems provide a similar rational on the predominant routes of drug elimination and the potential effect of transporters on (hepatic) drug disposition as illustrated in Fig. 6. Yet, assignment into BDDCS relies on clinical elimination information and is therefore not really applicable for early Drug Development. 


\section{ECC and IVIVE}

The correlation between the in vitro predicted $\left(\mathrm{CL}_{h, \text { pred }}\right)$ and in vivo observed $\left(\mathrm{CL}_{\mathrm{h}, \mathrm{obs}}\right)$ hepatic clearances for our dataset based on the mechanistic in vitro-in vivo extrapolation (IVIVE) method represented by Eq. (1) and Eq. (2) is illustrated in Fig. 7 (panel A). The approach reveals an excellent correlation with 11 out of 18 drugs predicted within two-fold deviation from the clinically observed value. The prediction accuracy in terms of average fold error (afe) and geometric mean fold error (gmfe) was 0.92 and 1.55, respectively. Present results demonstrate that the extended clearance concept model is by far exceeding the accuracy and performance of other IVIVE prediction methods for hepatic clearance based only on in vitro metabolism (Fig. 7, panels B and C for microsomes ( $\mathrm{afe}=0.76$, gmfe $=2.11$ )) and hepatocytes (afe $=0.32$, gmfe $=3.56)$, respectively) or sinusoidal uptake (Fig. 7, panel $D$, afe $=1.71$, gmfe $=1.84)$ data.
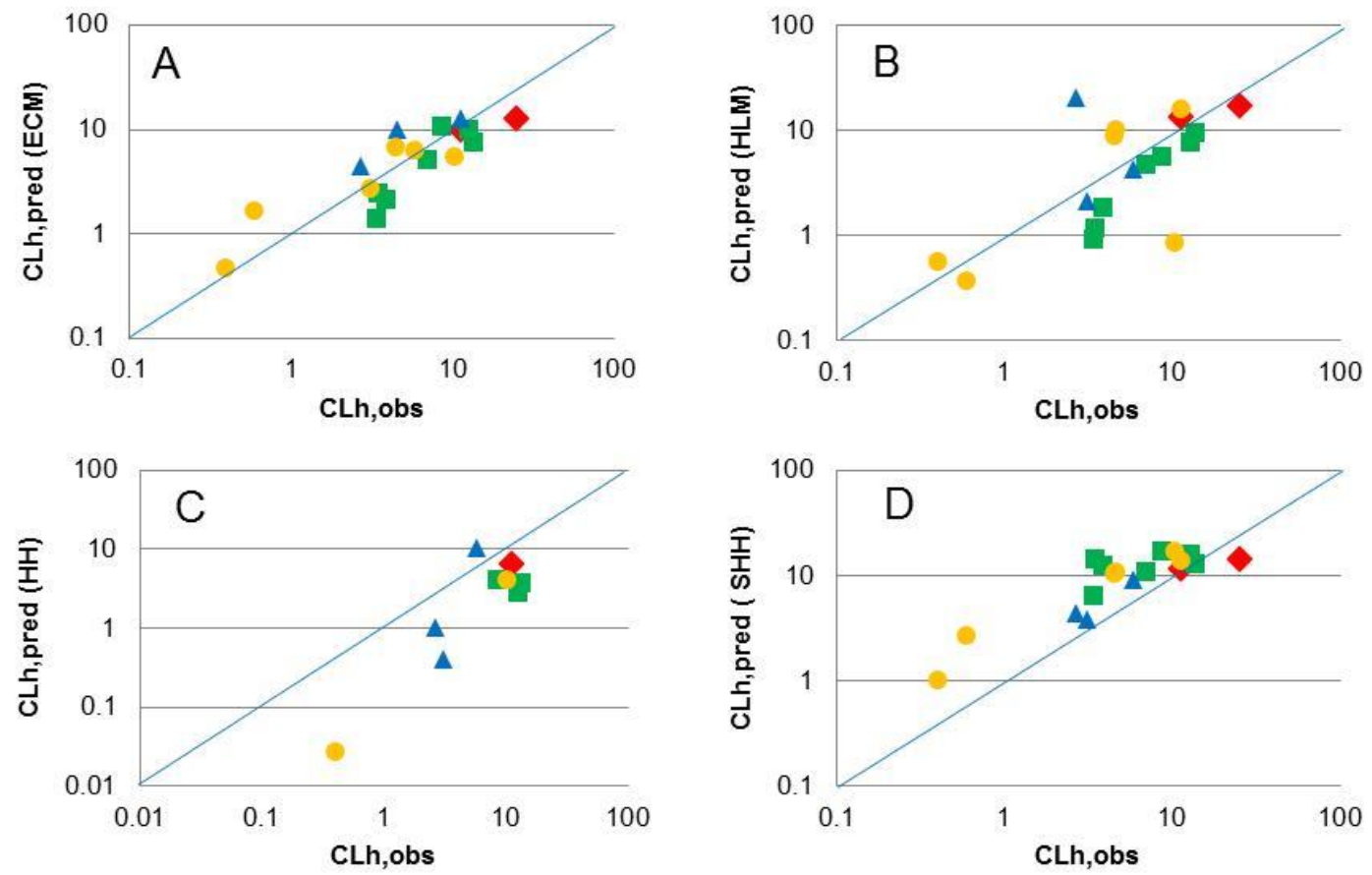

Figure 7. Comparison of the reported $\left(C L_{h, o b s}\right)$ and predicted $\left(C L_{h, \text { pred }}\right)$ hepatic clearances using different IVIVE methods. Panel $\mathbf{A}$ represents the relationship according to the extended clearance model (ECM). The panels $\mathbf{B}$ and $\mathbf{C}$ show the predictions based on in vitro metabolism data from human microsomes (HLM) and hepatocytes (HH), respectively, whereas panel $\mathbf{D}$ is the representation based on sinusoidal uptake data from suspended human hepatocytes (SHH). Red diamonds, green squares, blue triangles and orange circles show the class 1, 2, 3 and 4 assignments according to ECC (Table 1). The blue line represents the line of unity for each panel.

In accordance with previous research, the correlation analysis in Fig. 7 reveals a systematic underprediction of in vivo hepatic clearance when using metabolic turn-over data from human hepatocyte incubations whereas sinusoidal uptake data from suspended human hepatocytes tend to over-predict the in vivo situation $[9,10]$. Metabolic clearance data from human liver microsomes on the other hand are highly scattered along the line of unity providing under- and over-estimations of the observed human hepatic clearance. The potential rate-determining role of the hepatocyte membrane (missing in the microsomal system) is extensively discussed in literature. In line with this theory more in-depth data examination reveals that metabolism information from microsomes and hepatocytes generally provide reasonable results for the ECC class 1 and 2 compounds while hepatocyte uptake data seem to be highly predictive for the in vivo hepatic clearance of ECC class 3 compounds. IVIVE based on the extended mechanistic model surely works best for ECC class 4 compounds though. Imposing these observations on 
the principles of ECC the expected performance of the different IVIVE approaches can be summarized as depicted in Fig. 8. It is self-explanatory that with increasing contribution of renal clearance the predictability of all hepatobiliary IVIVE tools for overall (total) clearance is decreasing (tendency for underestimation). Based on above discussion, this is mainly true for ECC class 3 and 4 compounds while the overall prediction performance for ECC class 1 and 2 compounds likely remains good. In-house research intending to improve bottom-up predictions for overall human clearance using different in vitro and in vivo approaches is currently ongoing.

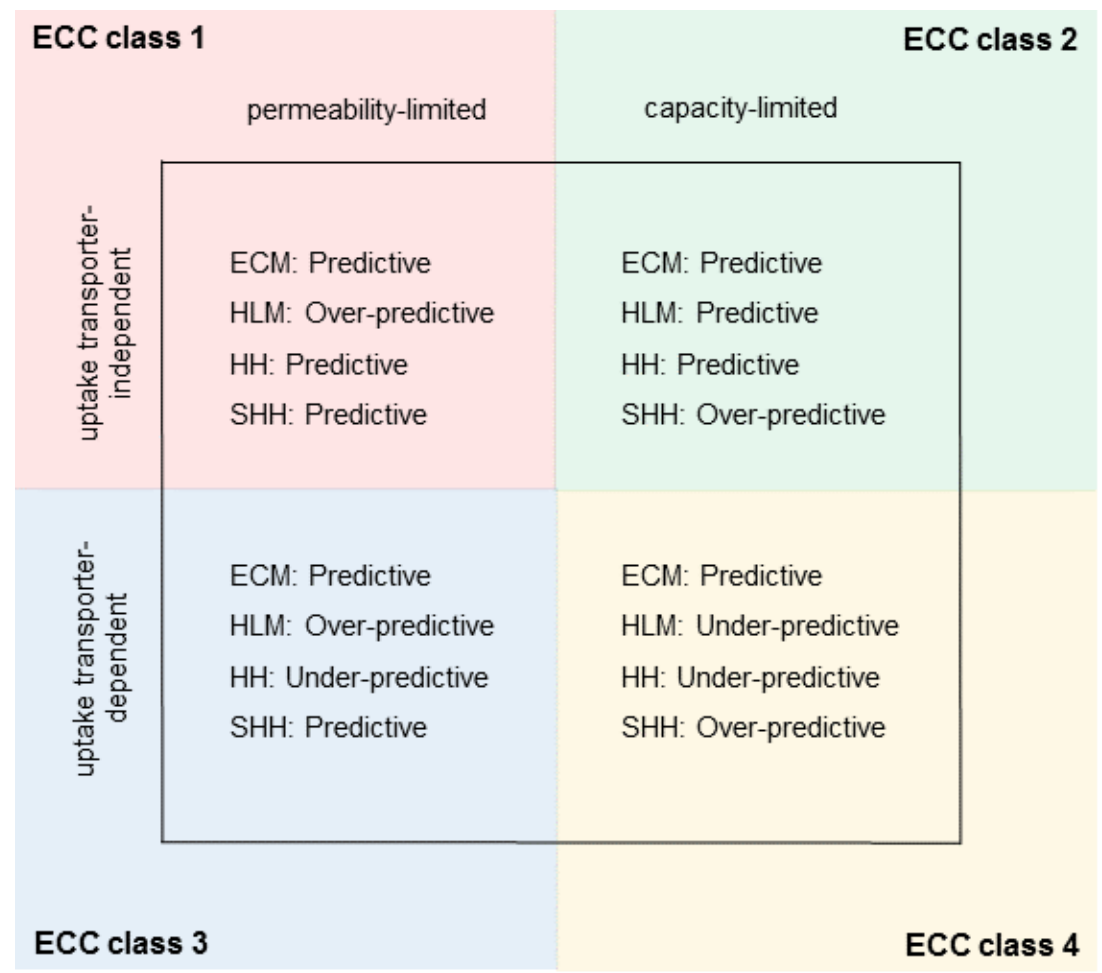

Figure 8. Anticipated hepatobiliary IVIVE accuracy of different in vitro tools: Metabolism assessment in human liver microsomes (HLM) or human hepatocytes $(\mathrm{HH})$, uptake data from suspended human hepatocytes (SHH) and integrated approach with the extended clearance model (ECM). For the assignment the following assumptions were made: (i) absence of significant biliary secretion (i.e. $\mathrm{CL}_{\text {int,sec }} \ll \mathrm{CL}_{\text {int,met }}$ ), (ii) absence of phase II metabolism and, (iii) no or down-regulated transporter activity in $\mathrm{HH}$.

\section{Static DDI predictions}

A perpetrator drug may inhibit any active clearance pathway contributing to the total hepatic elimination of a substrate. Accordingly, based on Eq. (1) (assuming $P S_{\text {eff,act }}=0$ and $P S_{\text {eff,pas }}=P S_{\text {inf,pas }}$ ), the overall hepatic intrinsic clearance in the presence of a perpetrator $\left(\mathrm{CL}_{h, i n t, i}\right)$ can be expressed as follows $[1,7]$ :

$C L_{h, \text { int }, i}=\frac{\left[\left(1-f_{i, \text { inf }}\right) \cdot P S_{\text {inf }, \text { act }}+P S_{\text {inf }, \text { pas }}\right] \cdot\left[\left(1-f_{i, \text { met }}\right) \cdot C L_{\text {int }, \text { met }}+\left(1-f_{i, \text { sec }}\right) \cdot C L_{\text {int }, \text { sec }}\right]}{\left[P S_{\text {inf }, \text { as }}+\left(1-f_{i, \text { met }}\right) \cdot C L_{\text {int }, \text { met }}+\left(1-f_{i, \text { sec }}\right) \cdot C L_{\text {int }, \text { sec }}\right]}$

where $f_{\mathrm{i}, \text { inf }}, f_{\mathrm{i}, \text { sec }}$ and $f_{\mathrm{i} \text {,met }}$ denote the inhibited fractions of active influx, canalicular secretion, and metabolism, respectively. $A f_{i}$ value of zero thereby indicates no inhibition whereas a value of one refers to 
complete inhibition. Based on this relationship the hepatic clearance in the presence of any perpetrator compound $\left(\mathrm{CL}_{h, i}\right)$ can be anticipated in accordance with Eq. (2).

Following oral (po) administration of a drug and its perpetrator, assuming the presence of hepatic and a non-hepatic (e.g. renal) elimination pathways and that the perpetrator drug only affects active processes in the liver, the exposure $(A \cup C)$ fold-change (expressed as $A \cup C_{p o, i} / A \cup C_{p o}$ ) can be described as follows:

$\frac{A U C_{p o, i}}{A U C_{p o}}=\frac{F_{h, i}}{F_{h}} \cdot \frac{1}{f n_{h} \cdot C L_{h, i} / C L_{h}+1-f n_{h}}$

where, $F_{h}\left(=1-C L_{h} / Q_{h}\right)$ and $F_{h, i}\left(=1-C L_{h, i} / Q_{h}\right)$ are the fractions of the oral dose escaping hepatic first-pass in the absence and presence of a perpetrator, respectively.

Under the additional assumption that the liver is the only clearance organ (i.e. $\left.f n_{h}=1\right)$ Eq. (4) simplifies to:

$\frac{A U C_{p o, i}}{A U C_{p o}}=\frac{C L_{h, i n t}}{C L_{h, i n t, i}}$

In an abbreviated manner, based on Eq. 5 and previous discussion, the drug-drug interaction (DDI) potential for the four ECM cases in Fig. 1 can therefore be represented as shown in Fig. 9.

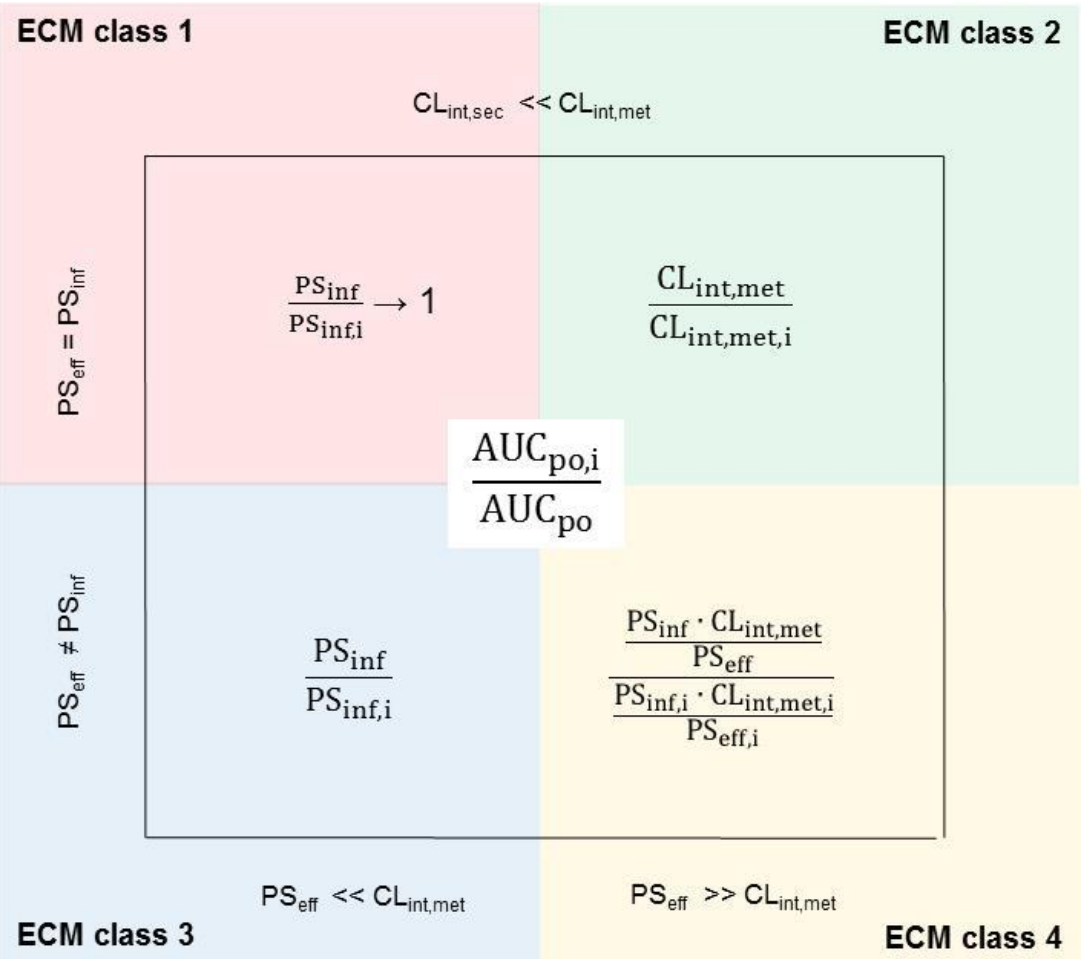

Figure 9. Compound class-dependent DDI prediction (inner panel) according to Eq. 5 for the four different ECM cases based on different pre-requisites (outer panel). i refers to a change in clearance in the presence of any perpetrator compound.

Fig. 10 depicts the predicted static DDI risk assessment of 3 representative compounds from our dataset (namely the ECM class 2 compound verapamil, digoxin as a typical ECM class 3 compound and pravastatin representing the ECM class 4) according to Eq. (4) and Eq. (5), assuming 90\% inhibition of the respective 
processes (i.e. $f_{\mathrm{i} \text {,inf }}=f_{\mathrm{i} \text {,met }}=f_{\mathrm{i} \text {, sec }}=0.9$, not taking into consideration fractional contributions of different enzymes or transporters to overall metabolic or transport clearance). In the absence of a renal clearance contribution (Fig. 10, panel A), in accordance with the extended clearance model, for the ECM class 2 compound in our selection, metabolism is identified as the major clearance mechanism primarily responsible for causing DDI's. Selective inhibition of active hepatic influx or biliary secretion has no significant effect on exposure though. A similarly distinct overall DDI behavior can be derived for the ECM class 3 compound digoxin with sinusoidal transporter inhibition being the major mechanism causing the interaction. For the ECM class 4 compound pravastatin, assuming absence of a renal clearance contribution, we predict a substantial AUC change upon concomitant inhibition of hepatic uptake, biliary secretion and metabolism whereas inhibition of the single clearance pathways results in comparatively moderate AUC ratios. Taking the clinically observed $f_{h}$ values according to Eq. (4) into account, the individual as well as the overall DDI risk assessment of verapamil is only marginally effected whereas the impact on the projected exposure changes for digoxin and pravastatin is significant (e.g. the overall $A \cup C_{p o, i} / A \cup C_{p o}$ ratio for pravastatin decreases about 8-fold) (Fig. 10, panel B). This concept is well reflected by ECM and can be rationalized by the process inter-dependencies as discussed above. It is noteworthy to mention at this point, that the extended clearance model represents the four extremes of hepatobiliary elimination and that most drugs settle somewhere in-between the ultimate limits given in Fig. 1. To eventually predict the overall DDI potential of drug molecules it is therefore essential to (quantitatively) assess all the individual hepatic process contributions as defined by Eq. (1).
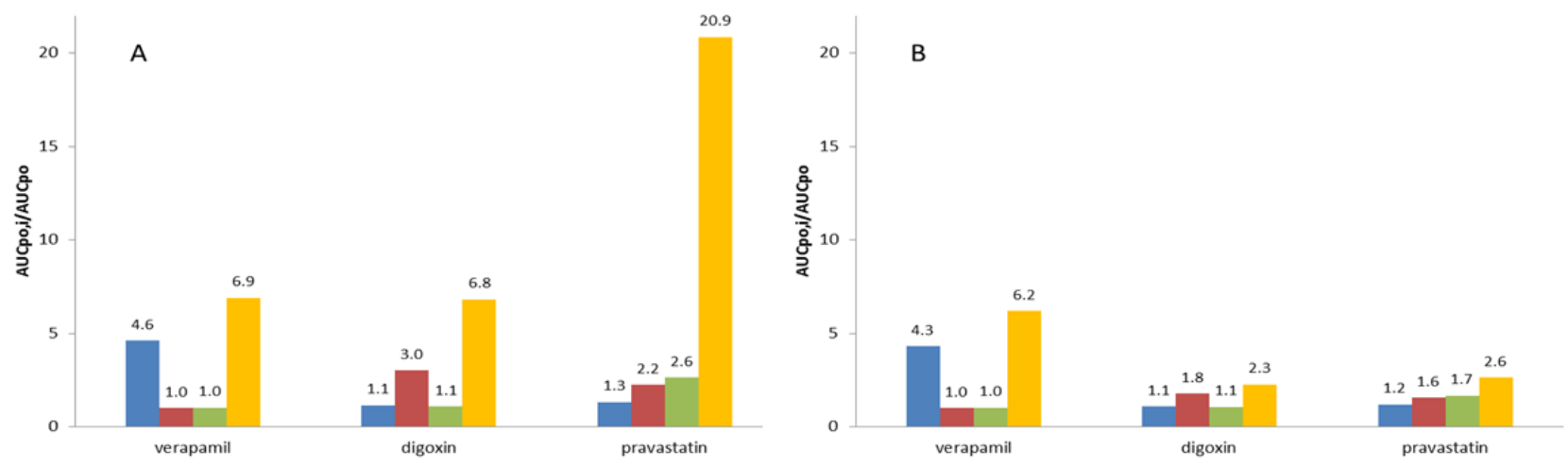

Figure 10. Static victim drug DDI predictions for verapamil (CYP2C8, CYP3A and P-gp), digoxin (P-gp, CYP3A and unknown sodium-dependent sinusoidal uptake transporter) and pravastatin (BCRP, MRP2, OATP1B1, OATP1B3, OATP2B1 and CYP3A4) according to Eq. (5) (panel A) and Eq. (4) (panel B). The projected AUC fold-changes with $\mathrm{f}_{\mathrm{i}, \mathrm{inf}}=$ $f_{i, \text { met }}=f_{i, s e c}=0.9$ are represented as follows: metabolism inhibition only (blue bars), exclusive sinusoidal active uptake inhibition (red bars), inhibition of canalicular efflux (light green bars) and, simultaneous inhibition of all active processes together (yellow bars).

Neglecting time-dependent concentration changes, static DDI predictions are expected to provide conservative (worst-case) estimates for the effective in vivo situation [1]. Nevertheless, DDI assessments according to Eq. (4) are generally in good agreement with clinical DDI data for ECM class 1 and 2 compounds as discussed elsewhere [7]. However, with increasing $f n_{\text {ren }}$ (i.e. decreasing $f n_{h}$ ), the predictions become less reliable for ECM class 3 and 4 compounds, often resulting in significant under-estimations of the effective DDI risk observed in clinics (e.g. pravastatin exposure in the presence of cyclosporine $A$ (inhibitor of OATP's, OAT's, NTCP, P-gp, BCRP, MRP2, CYP3A4 and UGT's) was reported to increase about 20-fold, significantly exceeding the theoretically possible hepatic DDI potential of 2.6-fold (Fig. 10)) [7]. The main reason for this observation is that a significant renal secretion process for these low permeable 
compounds is typically governed by active transporter processes which often are concomitantly inhibited by perpetrator drugs also interfering with the sinusoidal uptake carrier system in the liver. Consequently, for conservative bottom-up DDI estimates the physiologically less appropriate Eq. (5) should be preferred as it accommodates for possible cross-reactivity on hepatic and renal (transporters) systems (e.g. the clinical observation for pravastatin in the presence of cyclosporine A is well predicted with Eq. (5) (Fig. 10)). Nevertheless, in combination with clinical DDI data and applying a top-down approach, comparative static DDI assessments according to Eq. (4) and Eq. (5) can be extremely helpful in revealing alternative (extrahepatic) active elimination processes as discussed in full detail elsewhere [7].

\section{Prediction of unbound intracellular hepatic drug concentrations}

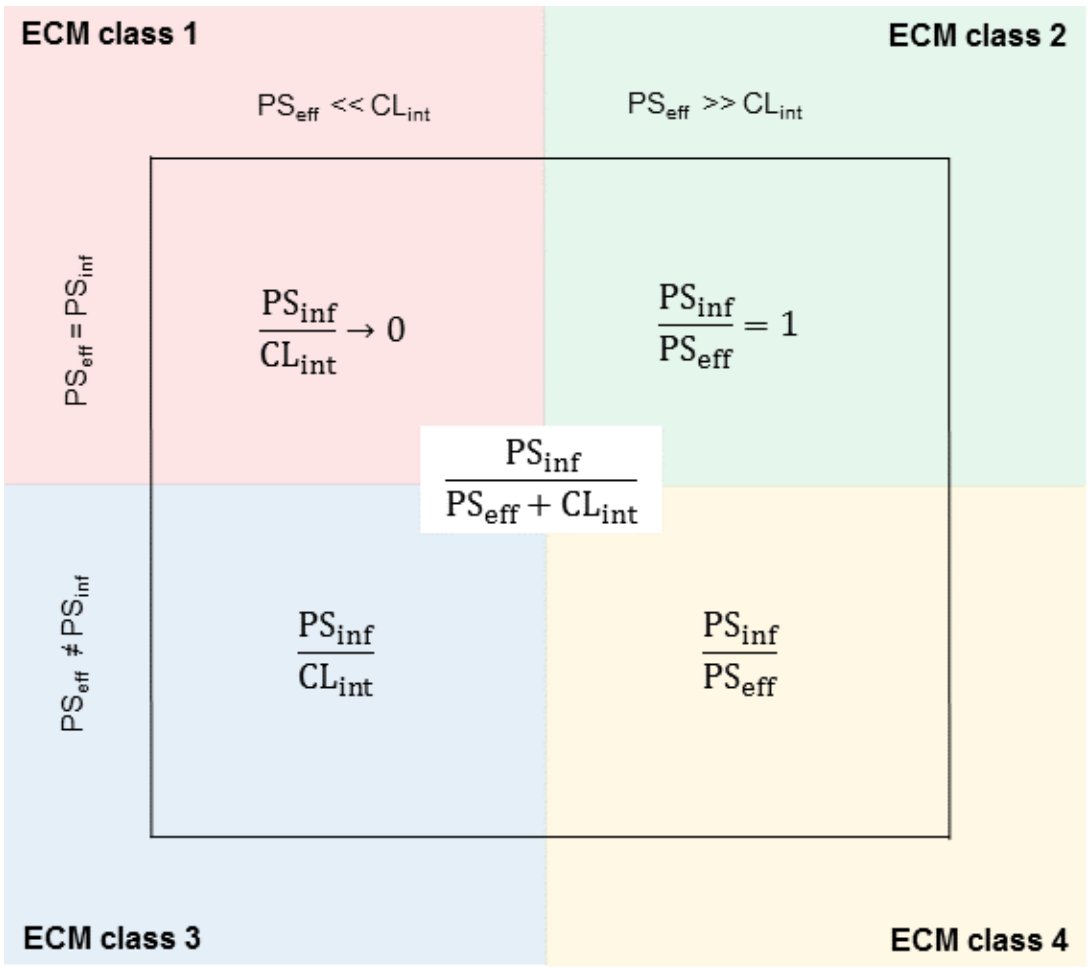

Figure 11. ECM class-dependent effect on the unbound liver-to-capillary blood concentration ratio ( $\left.\mathrm{Kp}_{\mathrm{uu}}\right)$ according to Eq. 6.

According to the so-called free drug hypothesis, at steady-state and in the absence of a membrane transport barrier limiting access to the hepatocyte, the unbound (free) intracellular concentration $C_{h, u}$ is equal to the unbound blood concentration $C_{b, u}$ (i.e. $C_{h, u} / C_{b, u}=1$ ). However, according to the extended clearance model, $\mathrm{C}_{\mathrm{h}, \mathrm{u}}$ is not only governed by uptake and protein binding but also by all intracellular elimination processes. Therefore, based on Eq. (1), for the liver this relationship needs to be rewritten as follows [11]:

$$
\frac{C_{h, u}}{C_{b, u}}=K p_{u u}=\frac{P S_{\text {inf }}}{P S_{e f f}+C L_{\text {int }}}
$$


where, $K \mathrm{p}_{\mathrm{uu}}$ is the unbound liver-to-capillary blood concentration ratio. In line with $\mathrm{ECM}$, the impact of the relative process contributions on $K \mathrm{p}_{\mathrm{uu}}$ can be summarized as shown in Fig. 11 . The knowledge of the relevant (effective) intrahepatic concentrations is important when performing DDI risk assessments (compound acting as inhibitor) on active processes such as metabolism and/or canalicular efflux. However, due to experimental inaccessibility of $C_{h, u}$, static perpetrator risk calculations for the liver generally refer to $C_{b, u}$ (usually expressed as $C_{b, u} / K_{i}$, where $K_{i}$ denotes the inhibition constant on a particular process). Based on the extended clearance model it becomes evident that the use of this substitute might lead to a considerable misjudgment of the DDI potential of perpetrator drugs though. Depending on the major ratedetermining process driving hepatic elimination significant under- (e.g. Kp $\mathrm{uu}_{\mathrm{uu}}$ equals 2.4 for ECM class 4 compound pravastatin) or over- (e.g. $C_{h, u}$ is about 80 -fold less than $C_{b, u}$ for ECM class 3 compound cimetidine) estimations of the real interaction risk might result. The use of systemic unbound drug concentrations (or unbound drug concentrations in the portal vein) is thus likely insufficient to properly assess the actual drug-drug interaction potential on intracellular enzymes/transporters for class 1, 3 and 4 compounds as illustrated in Table 2.

Table 2. Effect of $K p_{u u}$ on $C_{h, u}$ and the DDI risk outcome for perpetrator drugs.

\begin{tabular}{cll}
\hline ECM class & Effect of $\mathrm{Kp}_{\mathrm{uu}}$ on $\mathrm{C}_{\mathrm{h}, \mathrm{u}}$ (Eq. (6)) & Risk assessment with $\mathrm{C}_{\mathrm{b}, \mathrm{u}} / \mathrm{K}_{\mathrm{i}}$ \\
\hline 1 & $\mathrm{C}_{\mathrm{h}, \mathrm{u}}<<\mathrm{C}_{\mathrm{b}, \mathrm{u}}$ & overestimation \\
2 & $\mathrm{C}_{\mathrm{h}, \mathrm{u}}=\mathrm{C}_{\mathrm{b}, \mathrm{u}}$ & predictive \\
3 & $\mathrm{C}_{\mathrm{h}, \mathrm{u}}<$ or $>\mathrm{C}_{\mathrm{b}, \mathrm{u}}$ & under- or overestimation (generally overestimation as usually $\mathrm{PS}_{\text {inf }}<\mathrm{CL}_{\text {int }}$ ) \\
4 & $\mathrm{C}_{\mathrm{h}, \mathrm{u}}<$ or $>\mathrm{C}_{\mathrm{b}, \mathrm{u}}$ & under- or overestimation (generally underestimation as usually $\mathrm{PS}_{\mathrm{inf}}>\mathrm{PS}_{\mathrm{eff}}$ ) \\
\hline
\end{tabular}

Although currently not requested by health authorities present ECM-based principles might change the way we are doing perpetrator DDI risk assessments for intracellular hepatic processes in the future, all the more the mechanistic IVIVE method presented in this manuscript was demonstrated to provide quantitative reliable estimates of $\mathrm{CL}_{\mathrm{h}, \mathrm{obs}}$ and therewith of $\mathrm{Kp}_{\mathrm{uu}}$ (see above). Further in-house research on this this topic is currently ongoing.

\section{Conclusions}

In this review, we have illustrated the usefulness of the extended clearance model for classifying drug compounds depending on the relative contributions of the individual hepatic elimination processes. The ECM classification system based upon easily accessible in vitro data for metabolism, sinusoidal transport and canalicular efflux allows the quantitative bottom-up assessment of a series of (pharmacokinetic) parameters such as the hepatic clearance, the unbound liver-to-capillary blood concentration ratio and the static prediction of the DDI potential of drug molecules. The method reveals the major role of sinusoidal uptake as a gatekeeper for drug elimination, provides insight into the performance of different IVIVE approaches for hepatic clearance prediction and highlights potential limitations of currently applied drugdrug interaction risk assessment approaches. Consequently, following a thoughtful implementation, the approach may facilitate the compound selection process in Pharmaceutical Research and improve some of the compound profiling methodologies (e.g. DDI risk assessment) applied in Drug Development. 
Acknowledgements: The author wishes to acknowledge the many Novartis Drug Metabolism and Pharmacokinetic scientists of Basel, Switzerland, who have supported this work. Special thanks go to Dr Markus Zollinger for his critical evaluation of this manuscript.

\section{References}

[1] G. Camenisch and K. Umehara, Biopharmaceutics \& Drug Disposition 33 (2012) 179-1940.

[2] Y. Shitara, H. Sato and Y. Sugiyama, Annual Review of Pharmacology and Toxicology 45 (2005) 689723.

[3] K. Umehara and G. Camenisch, Pharmaceutical Research 29 (2012) 603-617.

[4] A. Kunze, B. Poller, J. Huwyler and G. Camenisch, Drug metabolism and drug interactions In Press.

[5] D. McGinnity, M. Soars, R. Urbanowicz and R. Riley, Drug Metabolism and Disposition 32 (2004) 1247-1253.

[6] D. Hallifax, E. Turlizzi, U. Zanelli and J. Houston, European Journal of Pharmaceutical Sciences 45 (2012) 570-574.

[7] A. Kunze, J. Huwyler, B. Poller, H. Gutmann and G. Camenisch, Journal of Pharmaceutical Sciences 103 (2014) 994-1001.

[8] L. Benet, G. Amidon, D. Barends, H. Lennernas, J. Polli, V. Shah, S. Stavchansky and L. Yu, Pharmaceutical Research 25 (2008) 483-48.

[9] M. Chiba, Y. Ishii and Y. Sugiyama, AAPS Journal 11 (2009) 262-276.

[10] D. Hallifax, H. Rawden, N. Hakooz and J. Houston, Drug Metabolism and Disposition 33 (2005) 1852-1858.

[11] Y. Shitara, K. Maeda, K. Ikejiri, K. Yoshida, T. Horie and Y. Sugiyama, Biopharmaceutics \& Drug Disposition 34 (2013) 45-78. 